http://www.jfas.info

\title{
FUZZY LOGIC SYSTEM FOR BBT BASED FERTILITY PREDICTION
}

\author{
M. S. M. Yazed ${ }^{1,2}$, F. Mahmud ${ }^{1,2, *}$ and M. Morsin ${ }^{1,2}$ \\ ${ }^{1}$ Faculty of Electrical and Electronic Engineering, Universiti Tun Hussein Onn Malaysia, \\ 86400 Parit Raja, Batu Pahat, Johor, Malaysia \\ ${ }^{2}$ Microelectronics and Nanotechnology Shamsuddin Research Centre (MiNT-SRC), \\ Universiti Tun Hussein Onn Malaysia, 86400 Parit Raja, Batu Pahat, Johor, Malaysia
}

Published online: 05 October 2017

\begin{abstract}
This paper introduce a fuzzy logic system for fertility prediction to improve the BBT technique based on FAM. The development of the fertility prediction system using fuzzy logic is motivated by its ability to conduct a complex relationships that exist between the input and its factors, which can address the issue of imprecision in the fertility prediction. According to the reference sets of BBT data tested on the fuzzy logic system for the prediction of ovulation and pregnancy, a significant result have been obtained with the accuracy of $95 \%$ and $80 \%$ respectively. Besides, this prediction system using fuzzy logic could improve the current practice in the FAM technique by integrating it with an Internet of Things (IoT) technology for automatic BBT charting and monitoring.
\end{abstract}

Keywords: family planning; fertility; BBT; fuzzy logic.

Author Correspondence, e-mail: farhanah@uthm.edu.my

doi: http://dx.doi.org/10.4314/jfas.v9i4s.27

\section{INTRODUCTION}

Most of the fertility awareness practitioner recommended Basal Body Temperature (BBT) measurement and charting technique for family planning because of its low-cost and safety 
advantages [1]. Basically, BBT is a condition where the body temperature is at lowest level, occurred during sleep state [2]. A normal woman BBT is between $33.2^{\circ} \mathrm{C}$ and $37.3{ }^{\circ} \mathrm{C}$, depending on the individual [3-4]. Generally, in the BBT measurement and charting technique, the user is required to plot and interpret her basal body temperatures in one women's menstrual cycle to obtain the progression in BBT graph. Moreover, in order to understand fertility of a woman, ones must know on how the technique of the BBT measurement and charting works.

Fertility of a woman can be characterized when ovulation and pregnancy happen inside the cycle which can be arranged through three stages; follicular stage, ovulation stage and luteal stage where the follicle stimulating hormones (FSH), luteinizing hormones (LH), estrogen and progesterone will roll out variations in the temperature diagramming. The follicular phase starts on the first day of menses until before ovulation phase. During this phase, basal body temperature will show a set of low temperature until the ovulation phase approaches. When ovulation phase comes, the estrogen hormones will get to a specific point of level, where the body realizes that the egg is developed and the time has come to ovulate. At this stage, a surge of LH will give the most astounding abatement in temperature that tells the egg is at long last prepared to be discharged. The arrival of the egg that is called ovulation can only last for around 24 hours. The body temperature will rise definitely after the ovulation comes. After the ovulation stage, the luteal period of the cycle starts. This stage is otherwise called "two week holds up", since it is the two weeks from when the ovulation happens until the beginning of another period or a pregnancy can be discovered. The body temperature stays high all through the luteal stage if pregnancy happened. From the above, it can be presumed that body temperature goes about as the fundamental sign in fertility regulation from the changing hormone level. Yet, the truth of the matter is that, the temperature only have a small change in its value [4]. Therefore, the device should be able to measure temperature with $0.01^{\circ} \mathrm{C}$ scale of accuracy.

Nowadays, the BBT measurement device has been sold widely in the market to assist woman in determining her fertility period. Yet, most of the commercial BBT devices cannot directly derive the information needed where the persons involved in the BBT measurement 
troublesomely need to plot their temperature readings manually into a BBT chart every day based on one BBT datum per day. They also need to interpret the changes in the BBT chart by themselves or to set several appointments with a medical practitioner for further consultation. The BBT conventional method to predict fertility by using charting on chart paper is known as observational method. Only few of the devices implement a convenient method in charting and monitoring the BBT which are not local based products. Besides, most of the products are using a statistical method to predict a fertility.

Statistical prediction method is the forecasting technique thought to be most suitable such as predicting the next fertile time or infertile time using the estimated values of the parameters within several months of data. Prediction by statistical method can also be described as the statistical inference. Indeed, a month of data can provide means of statistical knowledge about the data pattern to the whole data in a month for a cycle. Moreover, the observation statistical data has a close relationship with theoretical models such as probability distributions or the model used in regression analysis [9]. However, this method is typically dependent on system changes, where it cannot properly represent the complex relationships that exist between the input and the series of factors that influence it [5]. Consequently, this could cause a high tendency of wrong output prediction for ovulation. Therefore, this research will focus on developing a BBT prediction system with another approach from computational intelligent technology which is a fuzzy logic method that considers those complex relationships and complements the BBT prediction system.

Beginning of fuzzy logic was set apart in 1956, with the introduction of fuzzy set theory by Lotfi Aliasker Zadeh, he had provided a method to make definite decisions based on imprecise and ambiguous input data [6]. Fuzzy logic looks like human reasoning in its use of imprecise information to generate decisions by allowing subjective concepts of human such as very big and a long time to be expressed and mapped into exact numeric ranges. Thus, this makes it evolved into a very useful tool for solving complex real world problems. Nowadays, there are many medical areas that use the fuzzy logic method to compute and solve complex data such as pattern recognition in the evolution of patients, medical images of blood vessel, breast cancer and medical classification diagnosis [7]. The use of fuzzy logic provides 
advantage in medical area because basically, there is no need of mathematical models for mapping between inputs and outputs, but need to design the rules properly. This method will eventually help women in effectively predict their fertility based on automatic BBT charting and online monitoring concepts.

\section{METHODOLOGY}

In designing fertility prediction based on BBT using fuzzy logic, an investigation of fluffy rationale execution utilizing MATLAB Simulink has been directed for the expectation of ovulation and pregnancy in light of the BBT information. The fluffy rationale framework has been planned utilizing Fuzzy Logic Designer instrument from the MATLAB. The fuzzy system used in the inference engine is the Mamdani fuzzy system. For deffuzification, the input is set to centroid to obtain a crisp output. This prediction system will then be implemented in the Arduino microcontroller that is connected to the internet for the development of BBT device for automatic BBT charting and fertility prediction based on the an IoT application. The development of the fuzzy logic system can be defined using the following steps [8].

Step 1: Distinguish chief information, yield and process undertaking.

Step 2: Identify membership functions and define its parameter values.

Step 3: Use these membership functions and its parameter values to form procedural rules.

Step 4: Determine the defuzzification method.

Step 5: Test the system and modify if necessary.

\subsection{Fuzzy Ovulation Prediction System}

Ovulation prediction system is set to run in every cycle when there are more than one datum of BBT recorded. The latest BBT datum will be known as a dataAfter and the previous datum will be known as a dataBefore. These dataAfter and dataBefore will change from day to day depends on the BBT measurement and the ovulation prediction system will hold the highest different between the dataAfter and dataBefore in order to run in the ovulation prediction rules. Fig. 1 shows a block diagram of the fuzzy ovulation prediction system that consists of three 
inputs; the OvulationDay, MaxDelta and CycleLength, the constructed fuzzy ovulation rules based on several membership functions of the inputs and output, and the output of OvulationPrediction. OvulationDay is defined as the day of the ovulation happen, MaxDelta is defined as the maximum different between the dataAfter and dataBefore got from the held data, and CycleLength is defined as the length of a menstrual cycle obtained from a default value or from the previous cycle. The prediction system will run according to the rules that have been designed in order to predict the ovulation occurrence.

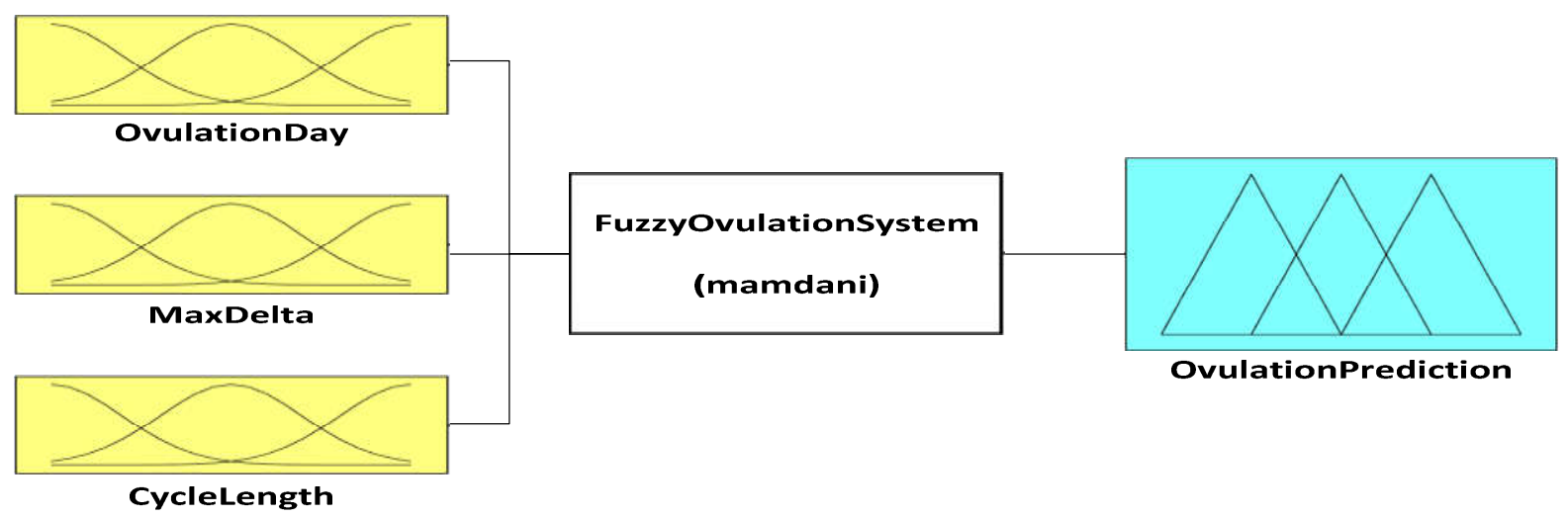

Fig.1. Block diagram of fuzzy ovulation prediction system

There are six membership functions (MFs) in the OvulationDay input, two MFs in the MaxDelta input, and four MFs in the CycleLength input. While in the output, there are two MFs. These MFs of the inputs and output with their parameter values are stated in the Table 1 according to the normal and abnormal ovulation day and cycle behaviour from the reference data.

Table 1. Inputs/output and membership function and its parameter value in fuzzy ovulation system

\begin{tabular}{|c|c|c|}
\hline Inputs/Output & Membership Function (MF) & MF Parameter Value \\
\hline \multirow{6}{*}{ OvulationDay } & $<6$ days & $=\left[\begin{array}{llll}-3.19 & 0 & 5 & 5.5\end{array}\right]$ \\
\hline & DayShort & $=\left[\begin{array}{llll}5.5 & 6 & 10 & 10.5\end{array}\right]$ \\
\hline & BSM & $=\left[\begin{array}{llll}10.5 & 11 & 14 & 14.5\end{array}\right]$ \\
\hline & DayMedium & $=\left[\begin{array}{llll}14.5 & 15 & 18 & 18.5\end{array}\right]$ \\
\hline & DayLong & $=\left[\begin{array}{llll}18.5 & 19 & 24 & 24.5\end{array}\right]$ \\
\hline & $>24$ days & $=\quad\left[\begin{array}{llll}24.5 & 25 & 58 & 58.5\end{array}\right]$ \\
\hline
\end{tabular}




\begin{tabular}{|c|c|c|}
\hline \multirow{2}{*}{ MaxDelta } & $<0.2$ & $=\left[\begin{array}{lllll}-0.9 & -0.1 & 0.1 & 0.23\end{array}\right]$ \\
\hline & $>0.2$ & $=\left[\begin{array}{lllll}0.2 & 0.89 & 1.09 & 1.9\end{array}\right]$ \\
\hline \multirow{4}{*}{ CycleLength } & Short & $=\left[\begin{array}{lllll}20.5 & 21 & 24 & 25.5\end{array}\right]$ \\
\hline & Medium & $=\left[\begin{array}{lllll}25.5 & 26 & 32 & 32.5\end{array}\right]$ \\
\hline & Long & $=\left[\begin{array}{llll}32.5 & 33 & 45 & 45.5\end{array}\right]$ \\
\hline & OverLong & $=\left[\begin{array}{llll}45.5 & 46 & 58 & 58.5\end{array}\right]$ \\
\hline \multirow{2}{*}{ OvulationPrediction } & NotOvulate & $=\left[\begin{array}{llll}-0.9 & -0.1 & 0.1 & 0.5\end{array}\right]$ \\
\hline & Ovulate & $=\left[\begin{array}{llll}0.5 & 0.6 & 1 & 2\end{array}\right]$ \\
\hline
\end{tabular}

*BSM is between short and medium

The important part in ovulation prediction is how to identify the ovulation occurrence. Fig. 2 shows the process flow of the ovulation prediction system. In order to identify the ovulation, the ovulation prediction system will calculate the difference between the dataAfter and the dataBefore $(\Delta \mathrm{D})$. The calculation value of the difference will be held in the EEPROM and the system will get the maximum difference between the dataAfter and the dataBefore (MaxDelta) as the input. While, the day of the dataBefore (which is the low temperature value) will be taken as the input. The MaxDelta, OvulationDay, and CycleLength inputs will be used into the ovulation fuzzy system to run ovulation prediction rules. The basic rule that has been constructed from the MFs in predicting the occurrence of ovulation is based on the MaxDelta is more than $0.2(>0.2)$ with the consideration of the women cycle length and the range of ovulation days. Basically, if MaxDelta is more than 0.2 there is a possibility that ovulation can occur. But, if the MaxDelta is less than 0.2 there will be no ovulation occur. 


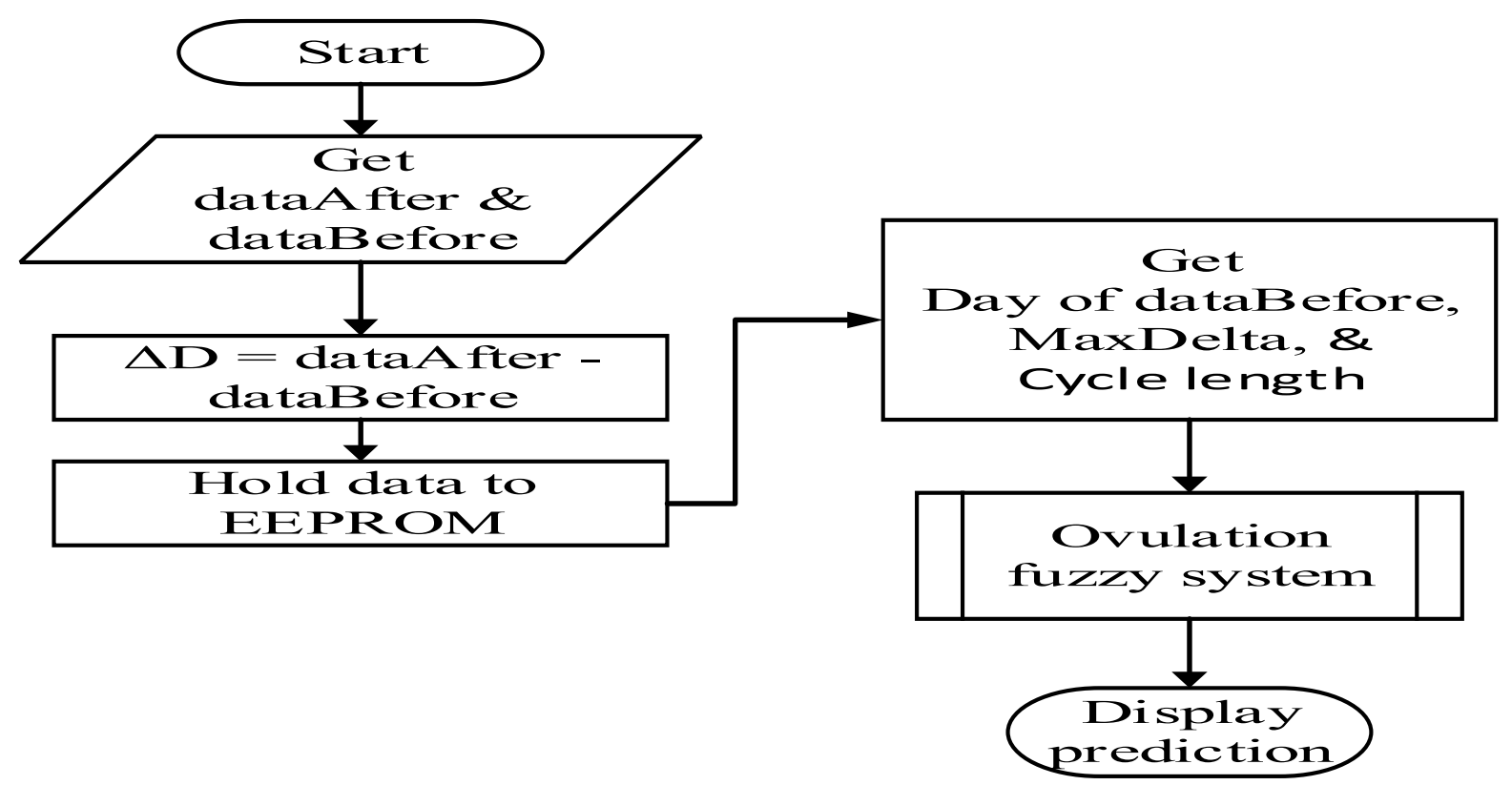

Fig.2. Process flow of ovulation prediction system

The surface view-relationship between OvulationDay and MaxDelta with the output of OvulationPrediction is as shown in Fig. 3, where ovulation will only occur in the range of 6 to more than 24 day and when MaxDelta is more than 0.2. Meanwhile, in Fig. 4, it shows that the ovulation day varies based on the cycle length where the range for Short, Medium, Long and OverLong are at around 21 to 25 days, 26 to 32 days, 33 to 45 days and more than 45 days respectively as shown in yellow colour. The yellow colour shows the area of ovulation that can happen. Here, the higher the value of the output, the higher the possibility of the ovulation to occur.

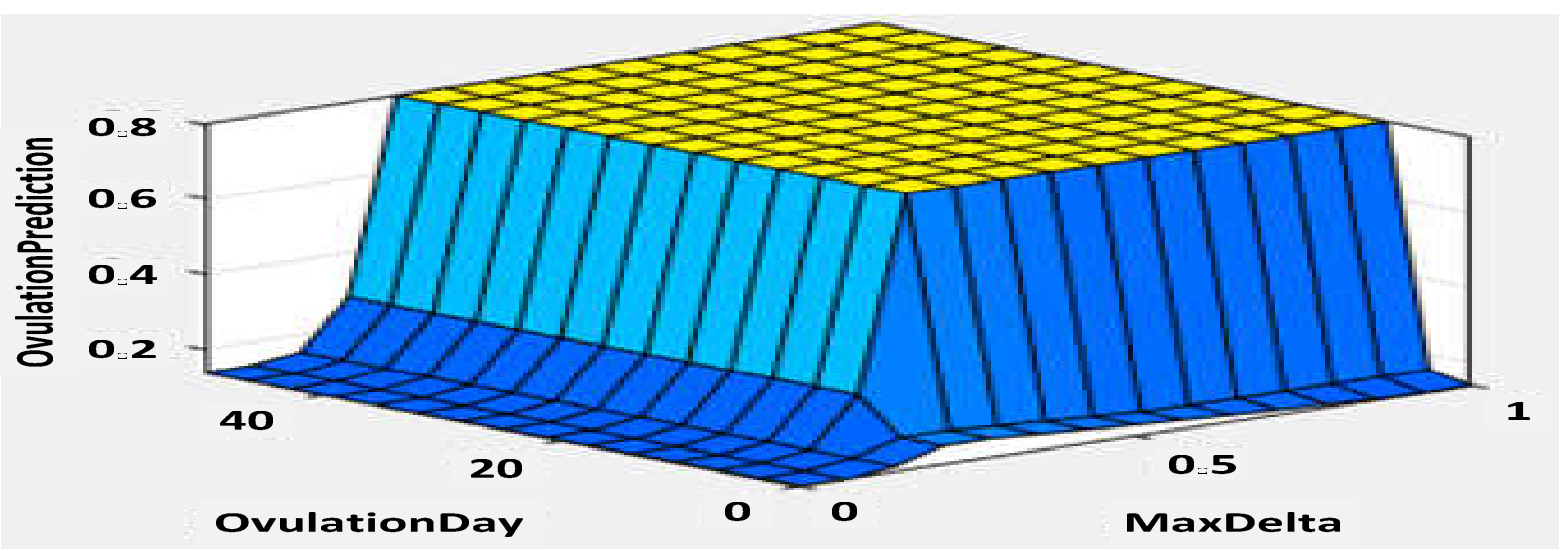

Fig.3. Surface view of inputs from OvulationDay and MaxDelta versus output of OvulationPrediction 


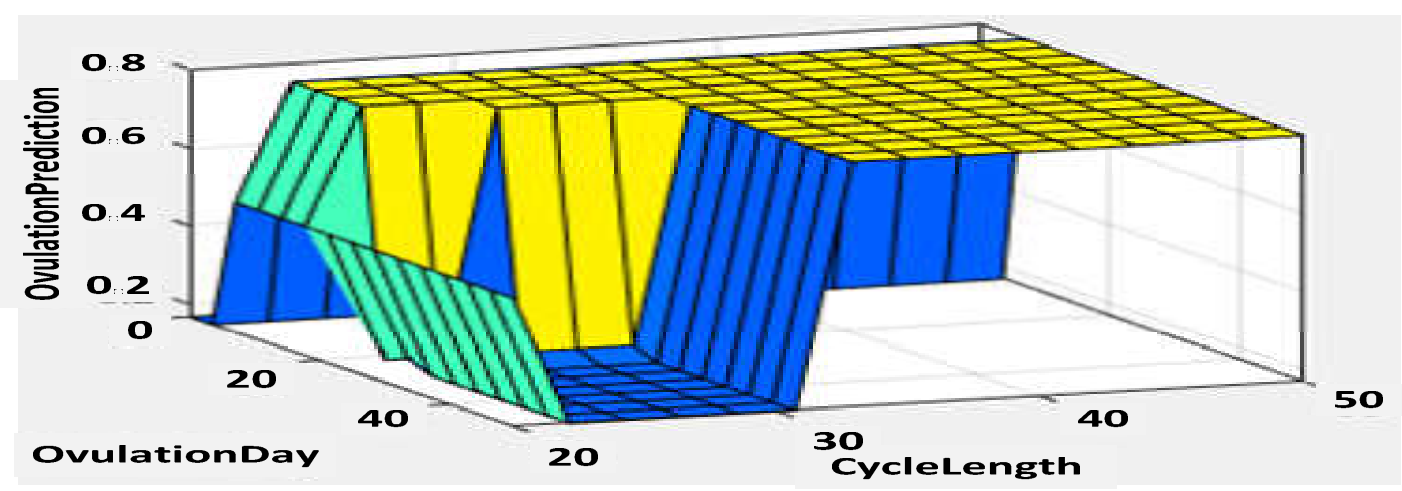

Fig.4. Surface view of inputs from OvulationDay and CycleLength versus output of

OvulationPrediction

\subsection{Fuzzy Pregnancy Prediction System}

Pregnancy prediction system is set to run every day after ovulation day is predicted. There are two inputs that need to be considered in predicting a pregnancy, which are the BBT data in the luteal phase and the length of days in the cycle. Fig. 5 shows the fuzzy pregnancy prediction system that consists of the inputs; DataRefertoMean and DayDifferent, the constructed fuzzy ovulation rules and the output of PregnancyStatus. DataRefertoMean is defined as the BBT data after the ovulation day whether the data condition is OverMean or UnderMean. If the data after ovulation day is over the mean, DataRefertoMean will receive value 1 that indicates yes; the data is OverMean. While, if the data after ovulation is under mean, DataRefertoMean will receive value 0 that indicates no; the data is UnderMean.

Besides DayDifferent is defined as the difference in the number of days between the current cycle length and the previous cycle length whether the data condition is OverDays or UnderDays. OverDays is when the current cycle length is overdue the previous cycle length in more than two days, while UnderDays can be defined as less than the OverDays. Basically, the system will run according to the designed rules in order to predict the pregnancy and the output of PregnancyStatus will be MaybePregnant only when the DataOverMean is OverMean and the DayDifferent is OverDays.

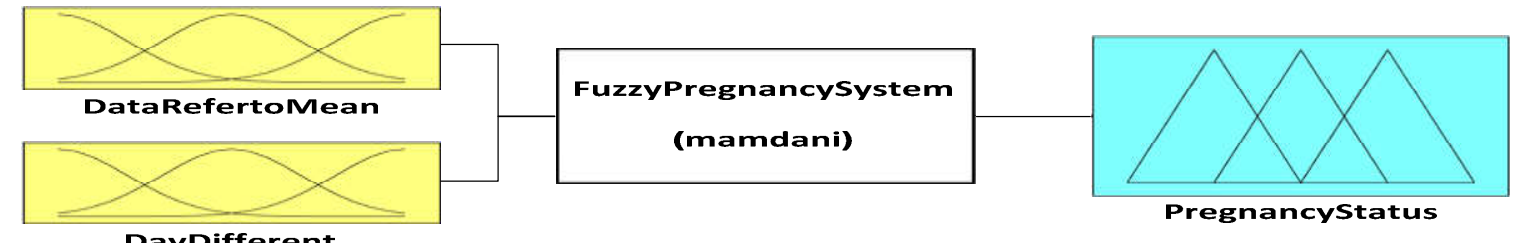

Fig.5. Block diagram of fuzzy pregnancy prediction system 
There are two membership functions (MFs) in the DataRefertoMean input, two MFs in the DayDifferent input and two MFs in the output. Table 2 shows the inputs, output and their MFs parameter values that need to be assigned by the temperature behaviour during pregnant.

Table 2. Inputs/output of membership function and its parameter value in fuzzy pregnancy system

\begin{tabular}{|c|c|c|}
\hline Inputs/Output & Membership Function (MF) & MF Parameter Value \\
\hline \multirow{2}{*}{ DataRefertoMean } & OverMean & $=\left[\begin{array}{lll}0.8 & 1 & 1.2\end{array}\right]$ \\
\hline & UnderMean & $=\left[\begin{array}{lll}-0.2 & 0 & 0.2\end{array}\right]$ \\
\hline \multirow{2}{*}{ DayDifferent } & OverDays & $=\left[\begin{array}{llll}0.5 & 3.4 & 17 & 34\end{array}\right]$ \\
\hline & UnderDays & $=\left[\begin{array}{lllll}-17 & -13.6 & -3.4 & 0.5\end{array}\right]$ \\
\hline \multirow[t]{2}{*}{ PrenancyStatus } & & $=\left[\begin{array}{lllll}0 & 0.2 & 0.4 & 0.5\end{array}\right]$ \\
\hline & Notr regnant Nayoer regnant & $=\left[\begin{array}{llll}0.5 & 0.6 & 0.9 & 1\end{array}\right]$ \\
\hline
\end{tabular}

Fig. 6 shows the process flow of the pregnancy prediction system. In order to predict the pregnancy, the pregnancy prediction system will retrieve the BBT data for day after ovulation, the previous cycle length and the current cycle length to gain the information on DataRefertoMean and DayDifferent. After the ovulation day has been predicted, in order to predict the pregnancy status, the system will refer to the DataRefertoMean and DayDifferent, and then will decide whether it is over or under the mean BBT from the previous cycle and whether it is shorter or longer than the previous cycle length based on the four conditions stated in Fig. 6. 


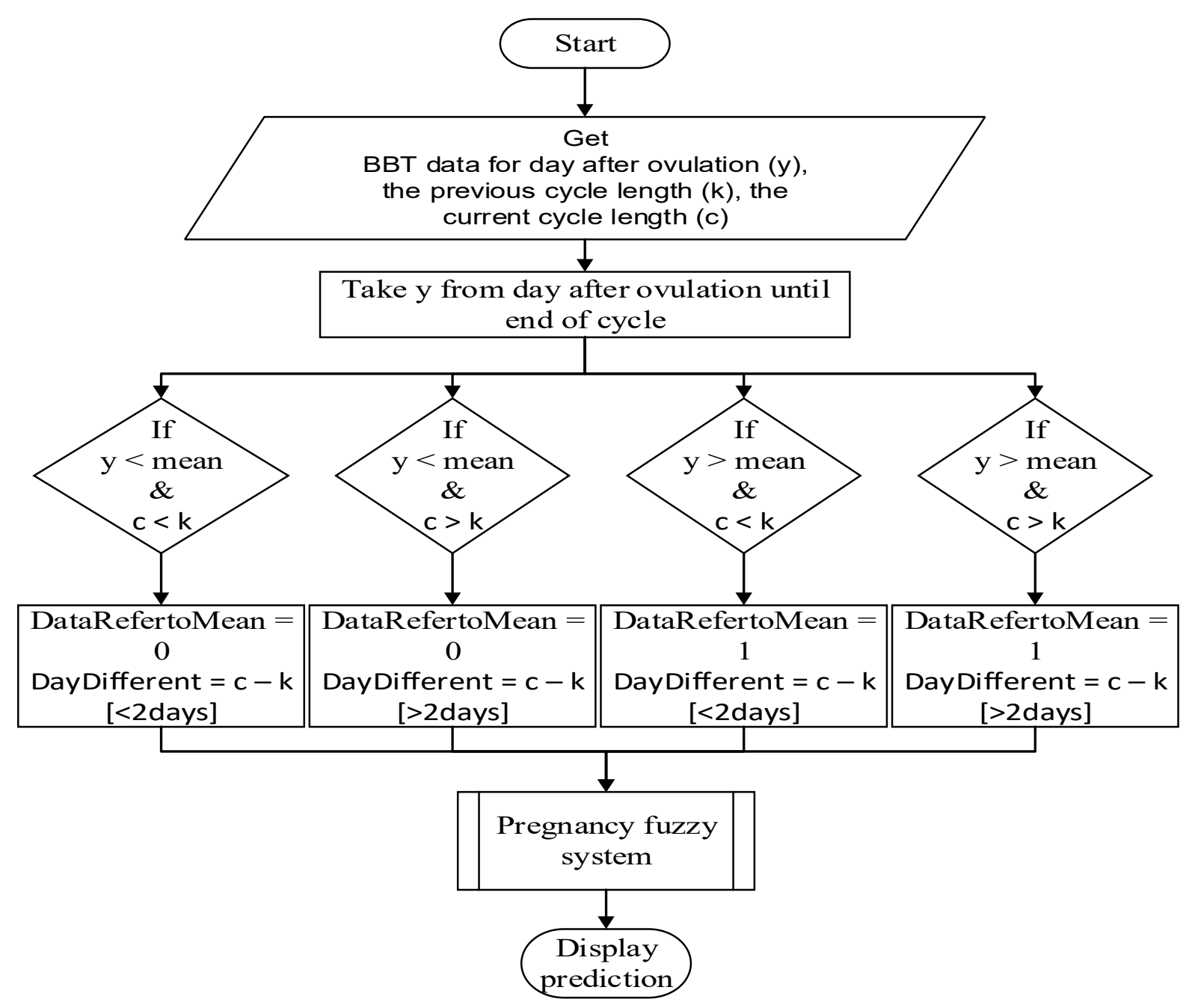

Fig.6. Process flow of pregnancy prediction system

Fig. 7 shows the surface view-relationship between DataRefertoMean and DayDifferent with the output PregnancyStatus of MaybePregnant or MaybeNotPregnant. Based on the output, it shows that pregnancy will only occur when OverMean and OverDays happen together. However, when there is only OverDays or only OverMean or UnderMean together with UnderDays occurs, then PregnancyStatus will be NotPregnant. Here, the higher the value of the output, the higher the possibility of the pregnancy to occur.

Meanwhile, in the case of a person having a fever, it should be noticed that the body temperature will not be reliable to be used for the fertility predictions since they should be based on BBT data without fever, Thus, a fever detection system also has been applied in this work to overcome the situation. 


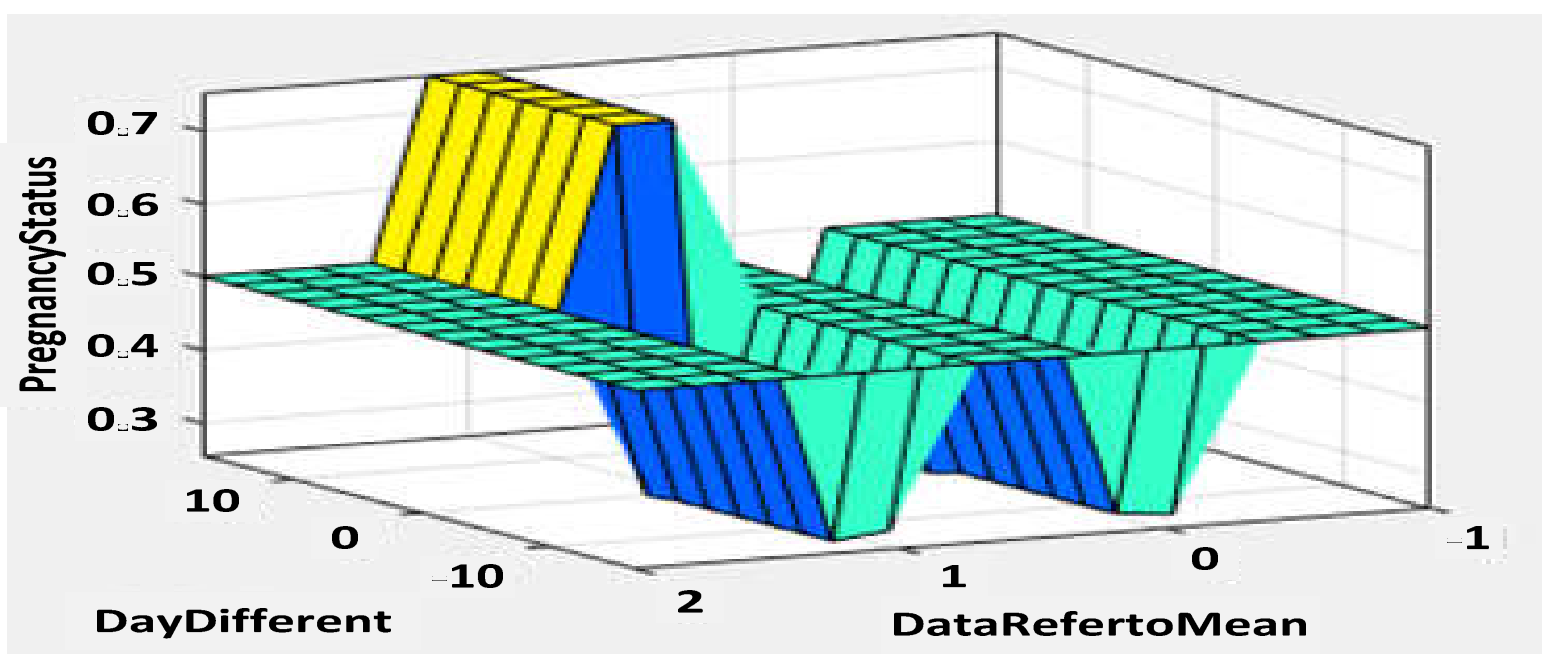

Fig.7. Surface view of inputs from DayDifferent and DataOverMean versus output of PregnancyStatus

\subsection{Fever Fuzzy Detection}

The fever fuzzy detection system is set to run every day when there is a new BBT data. This prediction is needed to make sure the BBT data value is at a normal body temperature without the influence of fever that could effect the reliability of the BBT charting. Fig. 8 shows the fever fuzzy detection system, which consists of two inputs from BBT and AmbTemp that refer to the body and ambient temperature respectively, the rules in FeverFuzzyDetection, and the output of Condition for NotFever and Fever.

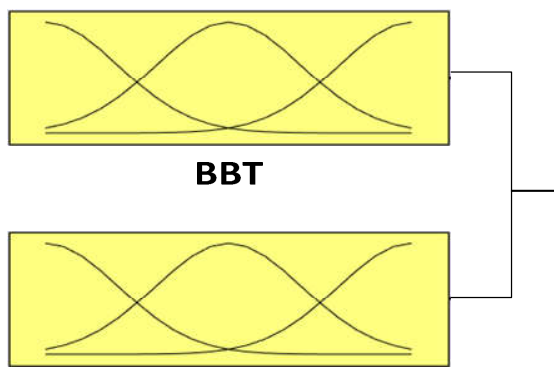

AmbTemp

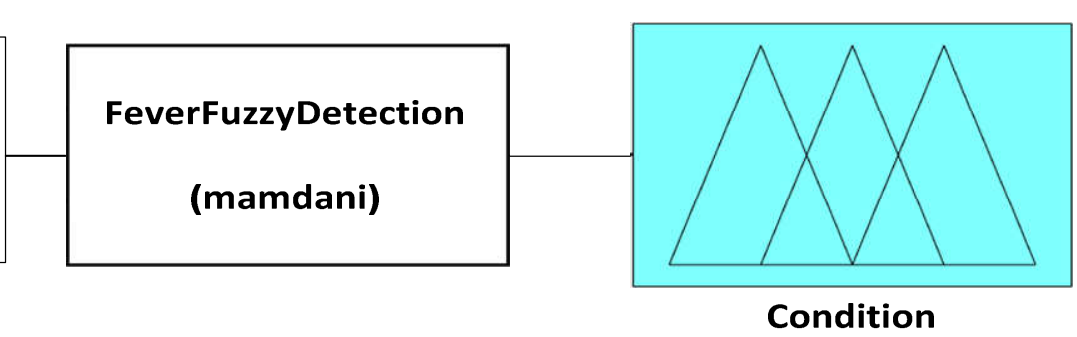

Fig.8. Block diagram of fever fuzzy detection system

There are two membership functions (MFs) in the BBT input, two MFs in the AmbTemp input and two MFs from the output. The details of the MFs from inputs and output with their MFs parameter values are stated in Table 3 according to the normal range of female body temperature. 
Table 3. Inputs/output membership function and its parameter value for fever fuzzy detection system

\begin{tabular}{|c|c|c|}
\hline Inputs/Output & Membership Function (MF) & MF Parameter Value \\
\hline \multirow{4}{*}{ BBT } & Normal & $=\left[\begin{array}{lllll}33 & 33.5 & 36.8 & 37.4\end{array}\right]$ \\
\hline & High & $=\left[\begin{array}{lllll}37.4 & 37.9 & 39.5 & 40\end{array}\right]$ \\
\hline & VeryCold & $=\quad\left[\begin{array}{lllll}16 & 16.5 & 18 & 18.5\end{array}\right]$ \\
\hline & Cold & $=\quad\left[\begin{array}{llll}18.5 & 19 & 24 & 24.5\end{array}\right]$ \\
\hline \multirow[t]{3}{*}{ AmbTemp } & $\mathrm{RT}$ & $=\quad\left[\begin{array}{llll}24.5 & 25 & 26.6 & 27.1\end{array}\right]$ \\
\hline & Hot & $=\quad\left[\begin{array}{lllll}27.1 & 27.6 & 34.5 & 35\end{array}\right]$ \\
\hline & VeryHot & $=\quad\left[\begin{array}{lllll}35 & 35.5 & 44.5 & 45\end{array}\right]$ \\
\hline \multirow[t]{2}{*}{ Condition } & NotFever & $=\left[\begin{array}{lllll}33 & 33.5 & 36.9 & 37.4\end{array}\right]$ \\
\hline & Fever & $=\left[\begin{array}{lllll}37.4 & 37.9 & 39.5 & 40\end{array}\right]$ \\
\hline
\end{tabular}

*RT: Room Temperature

According to the rules, there are only three conditions that indicate the person having a fever; when BBT is High and AmbTemp is at RT, when BBT is High and AmbTemp is Cold and when BBT is High and AmbTemp is VeryCold. When the person is detected in having a fever, the fever detection system will run a counter measure to react with the fever data and will get the approximate actual value of BBT based on Equation (1).

ActualData $=\frac{(\text { dataFever })+(\text { mean })}{2}$

The detection of fever can be realized by taking temperature measurement of body and ambient temperature at the same time as shown in Fig. 9. Then, based the designed fuzzy rules, the detection system will used them as the two input data to decide whether the BBT represents a fever or not. If it is represented the fever which is in the range of more than or equal to $37.4{ }^{\circ} \mathrm{C}$, the system will calculate the actual body temperature value using the average method to compensate the BBT datum. 


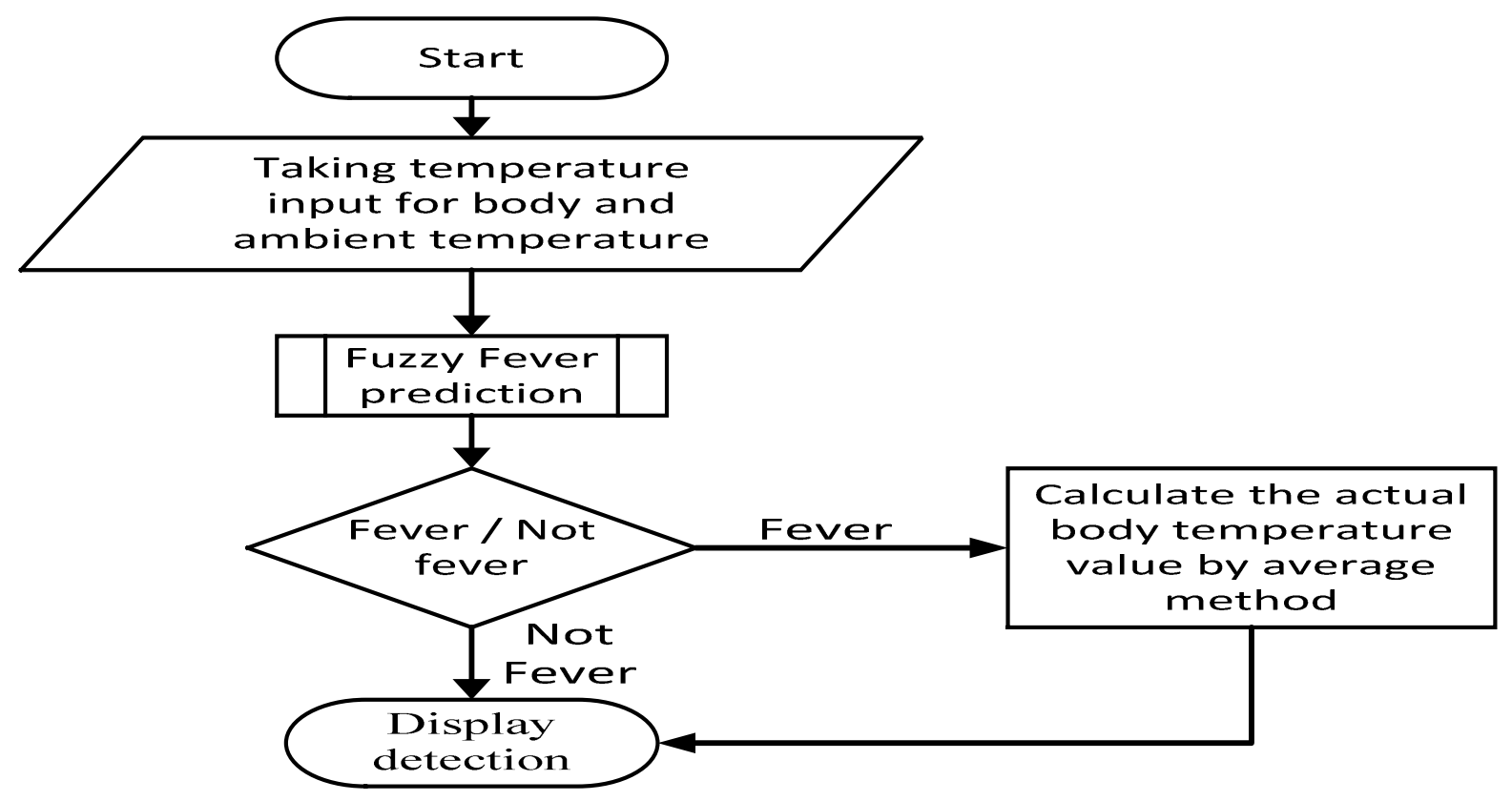

Fig.9. Block diagram of fever fuzzy detection system

Fig. 10 shows the surface view -relationship between the inputs from the BBT and the AmbTemp with the output of Condition of Not Fever and Fever. It shows that when the BBT and AmbTemp are both high, the condition is NotFever which shown as the peak surface in the view. This is because the rise in body temperature maybe affected by the high ambient temperature. But when the BBT measurement is high and AmbTemp measurement is low, the condition is Fever which shown as the peak in the view. Here, the higher the value of the output, the higher the possibility of the fever to occur.

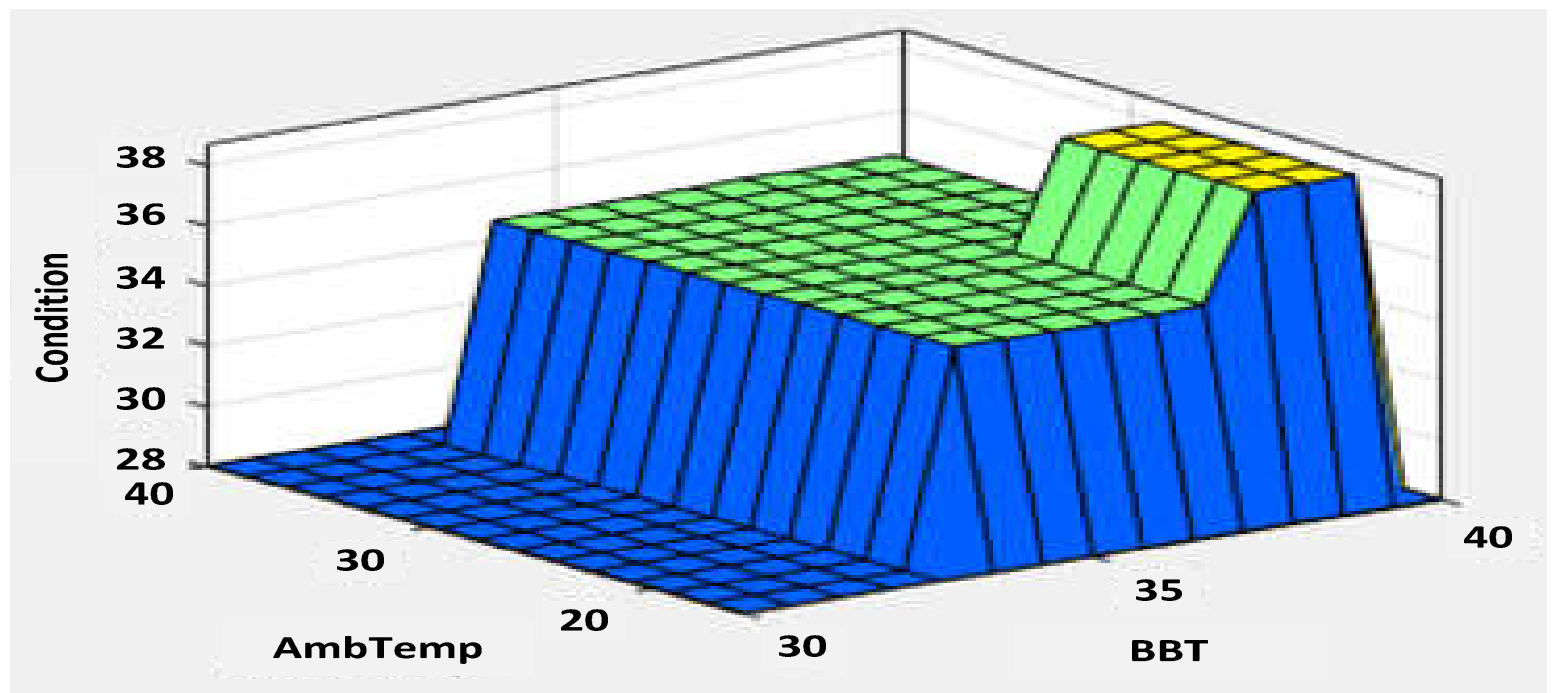

Fig.10. Surface view of inputs from BBT and AmbTemp versus output of Condition (Fever or NotFever) 


\section{RESULTS AND DISCUSSION}

Performance of a system is commonly evaluated using a confusion matrix technique. The confusion matrix has been done in order to test a system performance metric, which contains information about actual and predicted classifications result [13-15]. The fuzzy logic system in prediction for fertility based on BBT has been evaluated using this technique. Table 4 shows the example of confusion matrix for two class classifier. The entries in the confusion matrix have the following meaning in the context of this study.

- True Positive (TP) rate is the correct predictions number that an instance is positive.

- True Negative (TN) rate is the incorrect predictions number that an instance is negative.

- False Positive (FP) rate is the correct predictions number that an instance is negative.

- False Negative (FN) rate is the incorrect predictions number that an instance is positive.

Table 4. The example of confusion matrix for two class classifier

\begin{tabular}{ccc}
\hline $\mathbf{n}$ & Predicted: Positive & Predicted: Negative \\
\hline Actual: Positive & TP & FN \\
Actual: Negative & FP & TN \\
\hline
\end{tabular}

In this work, the performance of all the fuzzy systems which are fuzzy ovulation prediction system, fuzzy pregnancy prediction system and fever fuzzy detection system are evaluated by using the confusion matrix with 20 cycles of open source BBT data obtained from Fertility Friend website [12] and 77 BBT data from three cycles obtained from a healthy female subject aged 13 years old measured using the developed TempIoT 1.0 prototype [13]. The performance is evaluated based on accuracy parameter. Accuracy is the proportion of the total number of predictions that are correct and it is determined using Equation (2).

Accuracy $=\frac{(T P+T N)}{n} \times 100 \%$

\subsection{Fuzzy Ovulation System Performance Test}

Table 5 shows the confusion matrix table for fuzzy ovulation system evaluation using the 20 cycles of open source BBT data, $n=20$. From the table, the true positive is 19 and the false negative is 1 . While, false positive and true negative are 0 . This is because in the data there is no ovulation condition on NotOvulate. According to Equation (3), the accuracy of the fuzzy ovulation system is $95 \%$.

Table 5. Confusion matrix for fuzzy ovulation system 


\begin{tabular}{cccc}
\hline $\mathbf{n}=\mathbf{2 0}$ & Predicted: Positive & Predicted: Negative & \\
\hline Actual: Positive & $\mathrm{TP}=19$ & $\mathrm{FN}=1$ & 20 \\
Actual: Negative & $\mathrm{FP}=0$ & $\mathrm{TN}=0$ & 0 \\
& 19 & 1 & \\
\hline
\end{tabular}

Accuracy $=\frac{(19+0)}{20} \times 100 \%=95 \%$

\subsection{Fuzzy Pregnancy System Performance Test}

Table 6 shows the confusion matrix table for fuzzy pregnancy system evaluation using the 20 cycles of BBT data. From the table, the true positive is 6 and the false negative is 4 . While, false positive is 0 and true negative is 10. From the calculations in Equation (4), the accuracy of the fuzzy pregnancy system is $80 \%$.

Table 6. Confusion matrix for fuzzy pregnancy system

\begin{tabular}{cccc}
\hline $\mathbf{n}=\mathbf{2 0}$ & Predicted: Positive & Predicted: Negative & \\
\hline Actual: Positive & $\mathrm{TP}=6$ & $\mathrm{FN}=4$ & 10 \\
Actual: Negative & $\mathrm{FP}=0$ & $\mathrm{TN}=10$ & 10 \\
& 6 & 14 & \\
$\frac{(6+10)}{20} \times 100 \%=80 \%$ & &
\end{tabular}

\subsection{Fever Fuzzy Detection System Performance Test}

For the performance test of the fever fuzzy detection system, 77 BBT data from the three cycles are used. Table 7 shows the confusion matrix table for fever fuzzy detection system evaluation with only true negative results have been obtained since the subject is healthy and not having a fever during those cycles. Therefore, according to the performance evaluation represent by Equation (5), $100 \%$ accuracy of the system has been achieved.

Table 7. Confusion matrix for fever fuzzy detection

\begin{tabular}{cccc}
\hline $\mathbf{n}=\mathbf{7 7}$ & Predicted: Positive & Predicted: Negative & \\
\cline { 2 - 4 } Actual: Positive & $\mathrm{TP}=0$ & $\mathrm{FN}=0$ & 0 \\
Actual : Negative & $\mathrm{FP}=0$ & $\mathrm{TN}=77$ & 77 \\
Accuracy $=\frac{0+77}{77} \times 100 \%=100 \%$ & & & \\
\cline { 2 - 4 }
\end{tabular}




\section{CONCLUSION}

This work will be beneficial in improving the conventional FAM based on the BBT charting and monitoring technique through the prediction of ovulation and pregnancy [11] based on fuzzy logic [14]. Moreover, the developed fever detection system is also useful and yet important to ensure the reliability of the BBT data in predicting the fertility conditions. According to the performance evaluation based on the confusion matrix analysis, high percentage of accuracy has been achieved in all the systems. Therefore, in the near future, these systems will be implemented in an Arduino Yun Mini microcontroller to connect it with the Internet of Things (IoT) for the development of smart BBT monitoring device towards automatic BBT charting and high accuracy fertility prediction [10].

\section{ACKNOWLEDGEMENTS}

This paper is based upon fund supported by the UTHM under Grant Vote No. U460, Research and Innovation Fund and the MiNT-SRC, UTHM in providing laboratory facilities to complete the research.

\section{REFERENCES}

[1] Mbizvo M T, Phillips S J. Family planning: Choices and challenges for developing countries. Best Practice and Research Clinical Obstetrics and Gynaecology, 2014, 28(6):931-943

[2] Davie A, Amoore J. Best practice in the measurement of body temperature. Nursing Standard, 2010, 24(42):42-49

[3] Allen D. Determine ovulation with basal body temperature. Denver Nutrition, 2016, pp. $1-5$

[4] Kelly G. Body temperature variability (Part 1): A review of the history of body temperature and its variability due to site selection, biological rhythms, fitness, and aging. Alternative Medicine Review, 2006, 11(4):278-293

[5] Islam B U. Comparison of conventional and modern load forecasting techniques based on artificial intelligence and expert systems. International Journal of Computer Science Issues, 2011, 8(3):504-513

[6] Seising R. When computer science emerged and fuzzy sets appeared: The contributions 
of Lotfi A. Zadeh and other pioneers. IEEE Systems, Man, and Cybernetics Magazine, 2015, $1(3): 36-53$

[7] Barro S., Marín R. Fuzzy logic in medicine. Heidelberg: Physica-Verlag, 2013

[8] Akerkar R, Sajja P, Ashique R. Chapter 5-Fuzzy logic. 2006, https://www.slideshare.net/AshiqueRasool/chapter-5-27239629

[9] Florence K. Logistic regression and kernelized SVM. 2014, pp. 1-5

[10] Mawale M M, Chavan V. Implementation and simulation of fuzzy logic controllers for productivity and fertility of soil and performance evaluation of triangular membership function. Compusoft, 2014, 3(9):1098-1102

[11] Moreira M W, Rodrigues J J, Oliveira A M, Saleem K, Neto A. Performance evaluation of predictive classifiers for pregnancy care. In IEEE Global Communications Conference, 2016, pp. 1-6

[12] Tamtris Web Services Inc. FertilityFriend.com's chart gallery. 2017, https://www.fertilityfriend.com/cg/

[13] Yazed M S, Mahmud F. The development of IoT based BBT charting and monitoring using ThingSpeak. AIP Conference Proceedings, 2017, 1788(1):4-6

[14] Latip M F, Udin M K, Othman M M, Yassin I M, Rizman Z I, Zaini N, Hidayat M N, Aminuddin N, Herman S H, Saad H, Rahiman M H. Implementation of fuzzy logic-based final year project student-supervisor matching system. International Journal of Advanced and Applied Sciences, 2017, 4(4):159-163

\section{How to cite this article:}

Yazed MSM, Mahmud F, Morsin M.Fuzzy logic system for bbt based fertility prediction. J. Fundam. Appl. Sci., 2017, 9(4S), 475-491. 